\title{
Consumo de ansiolíticos benzodiazepínicos durante a pandemia de COVID-19: um estudo remoto com estudantes universitários
}

Consumption of benzodiazepine anxiolytics during the COVID-19 pandemic: a remote study with university students

Consumo de ansiolíticos benzodiazepínicos durante la pandemia del COVID-19: un estudio a distancia con estudiantes universitarios

Bruna Andrade Fontes

fontesbruna27@gmail.com

Universidade Salvador (UNIFACS) - Brasil https://orcid.org/0000-0003-3893-627X

Pablo Mateus dos Santos Jacinto

pablojacintopsi@gmail.com

Universidade Federal da Bahia (UFBA) - Brasil https://orcid.org/0000-0002-4894-5893

Renan Vieira de Santana Rocha

renanvsr@gmail.com

Universidade Federal da Bahia (UFBA) - Brasil https://orcid.org/0000-0003-4981-2854

\begin{abstract}
RESUMO
Problemas associados às ansiedades e ao ciclo sono-vigília são costumeiros na população brasileira. Nesta pandemia, contudo, suspeita-se do possível aumento destes quadros, destacando-se a população de estudantes universitários brasileiros. O presente estudo intenta, assim, coletar e analisar dados acerca do consumo de ansiolíticos benzodiazepínicos por parte de jovens universitários, durante o período de pandemia da COVID-19, possibilitando a construção de um perfil epidemiológico focalizado desta população. Trata-se de um estudo do tipo Survey on-line, que teve como público-foco estudantes universitários baianos, que participaram voluntariamente da pesquisa. Os resultados indicam um aumento de $25 \%$ no número de estudantes universitários que começaram a fazer uso de ansiolíticos benzodiazepínicos durante a pandemia da COVID-19, e que $8 \%$ destes fazem automedicação, evidenciando a necessidade de ações em saúde por parte das instituições de ensino superior que, se negligenciadas, podem resultar em problemas como adoecimento mental, conflitos nas relações interpessoais e evasão do ambiente universitário.
\end{abstract}

Palavras-chave: Ansiolíticos. Benzodiazepínicos. Pandemia. COVID-19. Saúde do Estudante.

\begin{abstract}
Problems associated with anxieties and the sleep-wake cycle are common in the Brazilian population. In this pandemic, however, a possible increase in these cases is suspected, especially the population of Brazilian university students. The present study therefore intends to collect and analyze data about the consumption of benzodiazepine anxiolytics by university students during the COVID-19 pandemic period, enabling the construction of a focused epidemiological profile of this population. This is an on-line Survey type study, whose target audience was university students from Bahia, who voluntarily participated in the research. The results indicate a $25 \%$ increase in the number of university students who started using benzodiazepine anxiolytics during the COVID-19 pandemic, and that 8\% of them selfmedicate, evidencing the need for health actions by educational institutions higher education that, if neglected, can result in problems such as mental illness, conflicts in interpersonal relationships and dropout from the university environment.
\end{abstract}

Keywords: Anxiolytics. Benzodiazepines. Pandemic. COVID-19. Student Health.

\section{RESUMEN}

Los problemas asociados con las ansiedades y el ciclo sueño-vigilia son comunes en la población brasileña. En esta pandemia, sin embargo, se sospecha un posible aumento de estos casos, especialmente en la población de estudiantes universitarios brasileños. Por lo tanto, el presente estudio tiene como objetivo recopilar y analizar datos sobre el consumo de ansiolíticos de benzodiacepinas por parte de estudiantes universitarios durante el período de pandemia de COVID-19, lo que permite construir un perfil epidemiológico enfocado a esta población. Este es un estudio de tipo 
Encuesta en línea, cuyo público objetivo fueron estudiantes universitarios de Bahía, que participaron voluntariamente en la encuesta. Los resultados indican un aumento del $25 \%$ en el número de estudiantes universitarios que iniciaron el uso de ansiolíticos benzodiazepínicos durante la pandemia de COVID-19, y que el $8 \%$ de ellos se automedican, evidenciando la necesidad de acciones sanitarias por parte de las instituciones educativas de educación superior que, de ser desatendidas, puede derivar en problemas como enfermedad mental, conflictos en las relaciones interpersonales y deserción del ámbito universitario.

Palabras Clave: Ansiolíticos. Benzodiazepinas. Pandemia. COVID-19. Salud Estudiantil.

\section{INTRODUÇÃO}

Em 11 de março de 2020, a Organização Mundial da Saúde (OMS) declarou estado de pandemia em decorrência da COVID-19, em escala global, causando grande impacto social, econômico e político ao redor do mundo (Fundação Oswaldo Cruz, 2020). Dentre as medidas tomadas para reduzir a contaminação da doença está o isolamento social, que acabou por exigir mudanças significativas, também, no ambiente acadêmico (Castioni, Melo, Nascimento \& Ramos, 2021).

Em razão do novo contexto de quarentena, as universidades passaram a se utilizar dos meios tecnológicos para manter a realização do cronograma das disciplinas, realizando suas atividades de forma remota (Maia \& Dias, 2020). Com isso, os estudantes precisaram também se adaptar, tanto ao modelo virtual de aulas, quanto ao isolamento e a todas as particularidades que tais mudanças impuseram (Castioni et al., 2021).

Essas questões se somam a outras tantas, que vão nos apontar mudanças também acerca do ambiente doméstico. Devido à pandemia do COVID-19 e seus desdobramentos, elencando-se o isolamento social como principal forma de prevenção da disseminação do vírus, várias mudanças ocorreram na rotina das pessoas ao redor do mundo, levando-nos, em muitos casos, ao que se tem chamado de "transição compulsória para o home office", fazendo com que muitos ambientes domésticos, então, tenham se tornado em ambiente de trabalho, acadêmico e de lazer, concomitantemente (Malta et al., 2020).

Frente a tal, focando-se a presente análise nas questões de maior âmbito acadêmico, o Ministério da Educação (MEC), na Portaria No. 343 de 17 de março de 2020, autorizou a substituição das disciplinas presenciais em cursos de graduação no Brasil por disciplinas remotas, mediante adequada adaptação, utilizando-se das Tecnologias de Informação e Comunicação (TICs) como principais ferramentas para tais substituições .

Qual o impacto dessa decisão na saúde mental de estudantes universitários brasileiros? Quadros comumente observados junto a esta população - como os das ansiedades e dos transtornos do sono (Ozamiz-Etxebarria, Dosil-Santamaria, Picaza-Gorrochategui \& Idoiaga-Mondragon, 2020) - podem ter se agravado em decorrência desta decisão? Os números associados ao uso de ansiolíticos benzodiazepínicos podem ser afetados em decorrência desta decisão? O que dizem os próprios estudantes universitários sobre esta situação, em termos de uma percepção de saúde autorreferida? Estas são as perguntas de investigação que orientam o presente estudo e que, a partir daqui, ensejaremos responder.

\section{FUNDAMENTAÇÃO TEÓRICA}

Ao pensar no contexto universitário atual, considera-se que tratamos de um momento no qual os estudantes universitários brasileiros estão passando por uma série de transformações, para 
além das responsabilidades e da pressão familiar à qual já estão costumeiramente submetidos (Silver, 1982 como citado em Ferreira et al., 2009). Podemos encontrar relevantes estudos, por exemplo, que avaliam os níveis de ansiedade de pessoas no contexto universitário, como o estudo desenvolvido por pesquisadores da Universidade José do Rosário Vellano (UNIFENAS), no sul de Minas Gerais, em que 50\% dos participantes (estudantes universitários) demonstraram algum sinal de ansiedade relacionado à vivência acadêmica (Carvalho, Junqueira, Cerdeira, Costa \& Santos, 2017). Assim, em razão da pandemia do novo coronavírus e da adaptação do espaço universitário para o ambiente virtual/doméstico, considera-se que existem vários novos desafios encontrados pela comunidade acadêmica e, destacadamente, para os estudantes universitários durante esse processo, que resultam em maiores chances de abandono das disciplinas, necessidade de supervisão dos processos de aprendizagem de forma mais efetiva e dificuldades na realização de atividades que eram costumeiramente presenciais no modelo remoto em decorrência do isolamento social obrigatório. (United Nations: Educational Scientific and Cultural Organization, 2020 como citado em Ferreira, Príncipe, Pereira, Oliveira \& Mota, 2020).

Outro exemplo das dificuldades sinalizadas até aqui pode ser encontrado em um estudo realizado no começo do período de pandemia, na Espanha, em que se procurava aferir os níveis de estresse, ansiedade e depressão em uma amostra populacional do norte do país. $\mathrm{O}$ estudo demonstrou que o número de aparição desses sintomas em pessoas mais jovens era consideravelmente maior do que para o restante dos participantes de outras faixas etárias. Segundo os autores, tais resultados, possivelmente, estão conectados ao impacto gerado pela necessidade de adaptação ao sistema remoto de ensino nas escolas e nas universidades (Ozamiz-Etxebarria et al., 2020) - o que pode ter relação paralela com o gradativo aumento do consumo de ansiolíticos que tem se observado, majoritariamente, nos últimos anos, junto ao público estudantil (Souza, Almeida, Amorim \& Santos, 2021).

Exemplo disto pode ver-se em um outro estudo, desenvolvido por pesquisadores da Universidade de São Paulo (USP) e realizado com estudantes de um curso de enfermagem, obtevese a informação de que $16 \%$ dos participantes estavam em uso ou já usaram algum ansiolítico ao longo da vida, e que $27 \%$ já aumentaram a dose sem consultar um médico - o que chamamos comumente de "automedicação" (Marchi, Bárbaro, Miasso \& Tirapelli, 2013). Isso demonstra não só taxas significativas do uso de medicamentos dessa classe, como também o perigo do manejo de doses sem orientação apropriada e, que em contextos de pandemia, podem estar acontecendo de forma mais incisiva - para além dos números já preocupantes de uso excessivo de ansiolíticos na população mundial e brasileira (Fávero, Sato \& Santiago, 2017).

Adicione-se aqui, ainda, dados de um estudo realizado por pesquisadores da Universidade Federal do Espírito Santo (UFES) com estudantes de graduação em medicina, que demonstrou que $13,1 \%$ da amostra em questão já havia consumido algum ansiolítico na vida, e que 1,8\% já havia feito consumo "pesado"; ou seja, mais de 20 vezes em um período de 30 dias. Os autores ainda mencionam a possibilidade desses números estarem relacionados com a extensa carga horária e com as responsabilidades advindas da universidade (Pereira, Souza, Buaiz \& Siqueira, 2008); o que, se ponderarmos ainda as especificidades das graduações das áreas da saúde, há, em estudo próximo, uma percepção autorreferida pelos estudantes de alta cobrança, o que poderia, na percepção deles, justificar esta relação com o alto consumo de ansiolíticos (Miranda et al., 2021).

Para além destes achados introdutórios, que intercalam nossos conhecimentos sobre os quadros associados às ansiedades, o uso de medicamentos psicotrópicos e a vivência universitária, há ainda estudos, que caminham em diversas direções, mas que mencionam relações entre sintomas ansiosos e distúrbios do sono - correlacionando e indicando causalidade entre os primeiros e os segundos - e evidenciando que um sono ruim pode colaborar no desenvolvimento de "estados de ansiedade". Essas variações no sono serão relatadas junto ao público constituído por estudantes, e podem estar relacionadas também às altas cargas horárias de estudos, por exemplo (Almondes \& Araújo, 2003). 
Todos estes fatores, apontados até aqui, evidenciam que o ambiente universitário já é, anteriormente, referido como "gerador de ansiedade", devido à sobrecarga que a multifuncionalidade exigida pelo mesmo pode gerar em diferentes entes das relações acadêmicas destacando-se, aqui, o alunado (Neri, Teston \& Araújo, 2020). A situação de isolamento e remodelagem que é demandada pela COVID-19 são fatores que, então, atrelados ao ambiente acadêmico, podem ter causado ou aumentado demandas emocionais e/ou psicossociais dos alunos e, consequentemente, tornado o consumo de medicamentos como ansiolíticos benzodiazepínicos mais propício - já que as ansiedades, como vimos, figurarão com profunda presença no padrão de consumo de medicamentos psicotrópicos nesta população estudada (Maia \& Dias, 2020). Doravante, em sendo possível observar estas relações, consideramos pertinente estudar o consumo de ansiolíticos benzodiazepínicos por parte desse público jovem - o que merece uma atenção especial, enquanto classe medicamentosa, por se tratar de medicamentos com alta taxa de dependência e alguns efeitos colaterais significativos associados - ponto em que justificamos o presente estudo.

Portanto, a presente pesquisa tem como objetivo geral coletar e analisar dados acerca do consumo de ansiolíticos benzodiazepínicos por parte de jovens universitários, durante o atual período de pandemia da COVID-19, possibilitando a construção de um perfil epidemiológico focalizado. É de extrema importância a discussão acerca dessa temática para que seja feito um mapeamento, além do consumo, das demandas que possam ter surgido ou se agravado durante este período, indicando-nos não apenas caminhos possíveis para a pesquisa, mas também para o delineamento de estratégias de cuidado eficazes para este público. Desta forma, procura-se também encontrar possíveis causas associadas ao uso desses medicamentos - tais como ansiedade, insônia e sofrimento por isolamento social - contextualizadas com o momento atual. A nomeação desses fatores permeia o contexto anterior à pandemia e podem ter sido agravados frente à atual situação pandêmica. Deste ponto, cremos que nossos resultados podem auxiliar a que sejam pensadas estratégias por parte das instituições de ensino superior (IES) para atender da forma mais adequada às necessidades dos alunos, intervindo junto a estes com o objetivo de minimizá-las, inclusive em termos das eventuais necessárias parcerias para a indicação de cuidados qualificados em saúde conforme se verá a seguir.

\section{PROCEDIMENTOS METODOLÓGICOS}

Trata-se de um estudo de levantamento (Survey on-line), com ênfase quantitativa, que teve como público-foco estudantes universitários do estado da Bahia, de instituições públicas e privadas, que responderam voluntariamente a um questionário on-line aberto e apresentado em quatro seções: $\left(1^{\mathrm{a}}\right)$ dados identificadores (semestralidade, curso, idade, gênero); ( $\left.2^{\mathrm{a}}\right)$ histórico diagnóstico e itinerário em saúde mental; $\left(3^{a}\right)$ sintomas e emoções experienciados durante a pandemia da COVID19; e ( $\left.4^{\mathrm{a}}\right)$ vivência acadêmica durante a pandemia.

A opção pelo método justifica-se pelo cenário pandêmico, bem como a familiaridade do público-alvo com as ferramentas virtuais devido ao ensino remoto emergencial. Segundo Gil (1989), em pesquisas sociais, tal método é adequado quando não há possibilidade de entrevistar todo o universo, e os comportamentos e características investigadas são facilmente acessadas e declaradas pelos participantes. Ademais, de acordo com Sue e Ritter (2007), em situações com limitação de tempo e restrição no acesso aos participantes, os levantamentos on-line são aplicáveis. Ademais, esse recurso facilita a tradução dos dados aos softwares estatísticos que sustentaram as análises realizadas.

O questionário on-line foi divulgado via redes sociais e ficou disponível para resposta durante os meses de novembro e dezembro de 2020, sendo a sua resposta opcional, anônima e mediante consentimento livre e esclarecido. A análise dos dados foi facilitada pelo software 
Microsoft Excel®, de modo a quantificar e identificar padrões de resposta, além de respostas excepcionais.

Não houve identificação de participantes sob quaisquer hipóteses, o que dispensou a submissão do mesmo a Comitê de Ética em Pesquisa (o que é habitual em pesquisas deste tipo); mas frisa-se que, a todo o tempo, o estudo tomou como referência ética a Resolução CNS $\mathrm{N}^{\circ}$. 510/2016, que versa sobre pesquisas com seres humanos em ciências sociais.

Os dados coletados foram tratados pelos pesquisadores conforme referenciais da pesquisa epidemiológica e estatística, sendo apresentados nos dois subtópicos a seguir.

\section{RESULTADOS E DISCUSSÃO}

O questionário on-line obteve cento e noventa e dois (192) respondentes da Bahia. Destes, $139(72,4 \%)$ do gênero feminino, $52(27 \%)$ do gênero masculino, e uma única pessoa se declarou não binária. Quanto à idade, $153(79,6 \%)$ estava na faixa entre 20 e 29 anos, seguido de $22(11,5 \%)$ entre 30 e 39 anos. Destaca-se que 92 (47,9\%) dos respondentes eram da área de Ciências Humanas, 61 (31,8\%) das Ciências da Saúde, e 23 (12\%) das Ciências Sociais Aplicadas . A maior parte se encontrava nos terceiro e quarto anos letivos, representando $47(24,5 \%)$ e $67(34,9 \%)$ da população entre o quinto e o oitavo semestres de curso.

A segunda seção de perguntas se referiu à trajetória de saúde mental dos respondentes. Destes, $47(24,5 \%)$ relataram ter algum diagnóstico psiquiátrico. A Tabela 1 apresenta os diagnósticos relatados.

\section{Tabela 1: Diagnósticos Psiquiátricos Apresentados pelas/os Respondentes}

\begin{tabular}{lccc}
\hline Diagnóstico & N & $\begin{array}{c}\text { Percentual em respondentes } \\
\text { com diagnóstico }\end{array}$ & $\begin{array}{c}\text { Percentual em total de } \\
\text { respondentes }\end{array}$ \\
\hline Transtornos de Ansiedade & 40 & 85,1 & 20,8 \\
Transtornos Depressivos & 26 & 55,3 & 13,5 \\
$\begin{array}{l}\text { Transtorno de Estresse Pós- } \\
\text { Traumático }\end{array}$ & 2 & 4,2 & 1 \\
Transtornos do Sono-Vigília & 2 & 4,2 & 1 \\
Outros & 7 & 14,9 & 3,6 \\
\hline
\end{tabular}

Fonte: Os autores (2021).

Observou-se um total de 48 respondentes que fazem uso de medicação, porém nem todos apresentam diagnóstico psiquiátrico. Dentre os respondentes com diagnóstico psiquiátrico, 16 (34\%) relataram não fazer uso de ansiolíticos, $11(23,4 \%)$ realizam uso de clonazepam e derivados (Nomes Comerciais: Rivotril, Epileptil, Clonasun, Zilepam, etc.), e 20 (42,6\%) sinalizaram utilizar outros medicamentos, não tendo especificado quais. Dos 17 respondentes sem diagnóstico psiquiátrico que fazem uso de medicação ansiolítica, $9(52,9 \%)$ utilizam clonazepam e derivados, 1 $(5,9 \%)$ utiliza midazolam e derivados, e $7(41,2 \%)$ outra medicação ansiolítica não especificada. 
Perguntou-se àqueles que utilizam medicação ansiolítica quando foi iniciado o uso. Destes, $36(75 \%)$ faziam uso anterior, enquanto $12(25 \%)$ começaram durante a pandemia da COVID-19. Por fim, os respondentes foram indagados sobre a prescrição, havendo $40(83,3 \%)$ que utilizavam medicação ansiolítica por indicação médica, $4(8,3 \%)$ adotavam a prática de automedicação, e 4 $(8,3 \%)$ receberam indicação de terceiros (o que, metodologicamente, também pode/deve ser definido como prática de automedicação).

A seção seguinte se referiu a experiências de emoções e sintomas vivenciados pelos respondentes durante a pandemia da COVID-19. A escolha desses elementos foi orientada pelas descrições do "Transtorno de ansiedade e correlatos" no DSM-5 (American Psychiatric Association, 2013). A Tabela 2 apresenta a distribuição de respostas por frequência.

Tabela 2: Emoções e Sintomas Experienciados durante a Pandemia

\begin{tabular}{|c|c|c|c|c|c|c|c|c|c|c|}
\hline \multirow{2}{*}{ Sintoma/Emoção } & \multicolumn{10}{|c|}{ Frequência } \\
\hline & 1 & $\%$ & 2 & $\%$ & 3 & $\%$ & 4 & $\%$ & 5 & $\%$ \\
\hline Calafrios & 98 & 51 & 49 & 25,5 & 31 & 16,1 & 13 & 6,8 & 1 & 0,5 \\
\hline Cansaço irregular & 13 & 6,8 & 24 & 12,5 & 53 & 27,6 & 66 & 34,4 & 36 & 18,8 \\
\hline Dificuldade em iniciar o sono & 21 & 10,9 & 31 & 16,1 & 46 & 24 & 50 & 26 & 44 & 22,9 \\
\hline Falta de ar & 73 & 38 & 39 & 20,3 & 52 & 27,1 & 27 & 14,1 & 1 & 0,5 \\
\hline Insegurança & 5 & 2,6 & 16 & 8,3 & 70 & 36,5 & 76 & 39,6 & 25 & 13 \\
\hline Insônia & 28 & 14,6 & 38 & 19,8 & 47 & 24,5 & 49 & 25,5 & 30 & 15,6 \\
\hline Medo iminente & 26 & 13,5 & 45 & 23,4 & 66 & 34,4 & 41 & 21,4 & 14 & 7,3 \\
\hline Menos horas de sono & 24 & 12,5 & 39 & 20,3 & 49 & 25,5 & 44 & 22,9 & 36 & 18,8 \\
\hline Nervosismo & 6 & 3,1 & 13 & 6,8 & 48 & 25 & 87 & 45,3 & 38 & 19,8 \\
\hline Palpitações irregulares & 43 & 22,4 & 46 & 24 & 61 & 31,8 & 36 & 18,8 & 6 & 3,1 \\
\hline Preocupação & 2 & 1 & 8 & 4,2 & 34 & 17,7 & 82 & 42,7 & 66 & 34,4 \\
\hline Sono interrompido & 23 & 12 & 40 & 20,8 & 50 & 26 & 58 & 30,2 & 21 & 10,9 \\
\hline Sonolência durante o dia & 8 & 4,2 & 25 & 13 & 50 & 26 & 82 & 42,7 & 27 & 14,1 \\
\hline
\end{tabular}

Legenda: $1=$ nunca $; 2$ raramente $; 3=$ ocasionalmente $; 4=$ frequentemente $; 5=$ a todo $o$ tempo.

Fonte: Os autores (2021).

A Tabela 2 demonstra a alta incidência de relatos acerca da alteração no processo de sonovigília, com índices elevados dos respondentes apresentando dificuldades em iniciar o sono $(48,9 \%)$, insônia $(41,1 \%)$, sonolência durante o dia $(56,8 \%)$, menos horas de sono $(41,7 \%)$ e sono interrompido $(41,1 \%)$ frequentemente ou a todo o tempo. 
Observou-se que os sintomas menos experienciados foram calafrios, falta de ar e palpitações irregulares. Dentre os sintomas com manifestações físicas, destaca-se o cansaço irregular, presente em 53,2\% dos respondentes em ocorrência frequente ou a todo o tempo. Houve alta incidência de experiência de medo iminente, nervosismo, insegurança e preocupação, as quais podem ser associadas ao desconhecimento permeado pela situação excepcional da pandemia.

A quarta e última seção, por sua vez, buscou identificar como os respondentes avaliam sua relação com o modelo de ensino remoto emergencial. As perguntas se referiram a aspectos importantes para a manutenção do rendimento acadêmico que podem ser influenciados pelo cenário da pandemia e pelas experiências relativas à saúde mental anteriormente abordadas. A Tabela 3 apresenta a sintetização dos dados obtidos através de uma escala de modelo Likert, na qual 1 representa o extremo negativo e 5 representa o extremo positivo. Vide:

Tabela 3: Experiências Acadêmicas durante a Pandemia

\begin{tabular}{|c|c|c|c|c|c|c|c|c|c|c|}
\hline \multirow{2}{*}{ Aspecto Acadêmico } & \multicolumn{10}{|c|}{ Resposta } \\
\hline & $\mathbf{1}$ & $\%$ & 2 & $\%$ & 3 & $\%$ & 4 & $\%$ & 5 & $\%$ \\
\hline Acompanhamento das aulas virtuais & 30 & 15,6 & 36 & 18,8 & 48 & 25 & 38 & 19,8 & 40 & 20,8 \\
\hline Adaptação ao modelo remoto & 38 & 19,8 & 50 & 26 & 47 & 24,5 & 28 & 14,6 & 29 & 15,1 \\
\hline Concentração durante aulas & 63 & 32,8 & 62 & 32,3 & 38 & 19,8 & 21 & 10,9 & 8 & 4,2 \\
\hline Motivação com as aulas & 91 & 47,4 & 50 & 26 & 31 & 16,1 & 8 & 4,2 & 12 & 6,3 \\
\hline Pensamento de trancamento do curso & 32 & 16,7 & 11 & 5,7 & 30 & 15,6 & 21 & 10,9 & 98 & 51 \\
\hline Queda no Rendimento acadêmico & 22 & 11,5 & 24 & 12,5 & 67 & 34,9 & 46 & 24 & 33 & 17,2 \\
\hline Sobrecarga de atividades acadêmicas & 11 & 5,7 & 24 & 12,5 & 38 & 19,8 & 51 & 26,6 & 68 & 35,4 \\
\hline
\end{tabular}

Legenda: 1 = extremo negativo; 5 = extremo positivo.

Fonte: Os autores (2021).

A Tabela 3 demonstra um quadro de alerta em relação à vivência acadêmica dos respondentes. Destes, $45,8 \%$ declararam estar com alta dificuldade de adaptação ao modelo remoto, $65,1 \%$ afirmaram não estar conseguindo se concentrar nas aulas e $61,9 \%$ pensam com alta frequência em trancar o curso. O rendimento acadêmico foi autoavaliado pelos estudantes respondentes, dos quais 34,9\% não declarou alteração devido ao modelo remoto emergencial, enquanto 41,2\% sinalizaram menor rendimento. Mesmo após dois semestres de ensino remoto, apenas 29,7\% dos respondentes afirmou estar se adaptando bem a esse formato; dado que pode se relacionar com a desmotivação com as aulas, expressa por $73,4 \%$ dos estudantes respondentes. Por fim, observou-se que o modelo remoto tem gerado sobrecarga para a maioria dos participantes, tendo aproximadamente $62 \%$ declarado alto índice nesse aspecto.

A análise dos dados obtidos na pesquisa gera um debate sobre três fatores importantes que envolvem a experiência dos estudantes respondentes: $\left(1^{\circ}\right)$ histórico de saúde mental e uso de 
medicamentos; $\left(2^{\circ}\right)$ emoções vivenciadas durante o período de pandemia; e $\left(3^{\circ}\right)$ grau de dificuldade com a adaptação ao modelo remoto de ensino dos universitários.

É de importância destacar, no que se refere ao histórico de saúde, o apontamento de diagnósticos de ansiedade e depressão entre $20,8 \%$ e $13,5 \%$ dos respondentes, respectivamente. Esses números seguem o padrão de outro estudo realizado com universitários da área de saúde no Nordeste do Brasil, em que a prevalência de depressão foi de $28,6 \%$ e ansiedade $36,1 \%$ (Leão, Gomes, Ferreira \& Cavalcanti, 2018). Essa pesquisa, somada às demais que apresentamos no início deste estudo, comprova que os elevados índices de questões relacionadas à saúde mental entre estudantes universitários estão diretamente ligados ao ambiente acadêmico, que pode ser um contexto favorável ao aparecimento de sinais e sintomas dessa ordem.

No que se refere ao consumo de benzodiazepínicos, o clonazepam aparece como medicamento mais consumido entre os respondentes, sejam com ou sem diagnóstico psiquiátrico. Isso evidencia o alto padrão de consumo desse psicotrópico, em que: "Considerando todas as formulações industrializadas e as manipuladas sujeitas a controle especial, ele foi o princípio ativo mais consumido no País, entre 2007 e 2010" (Agência Nacional de Vigilância Sanitária, 2016 como citado em Zorzanelli et al., 2019, p. 3130).

Outro dado obtido através da pesquisa evidencia 16,6\% de uso de ansiolíticos sem orientação médica, o que, segundo Castro, Mendes, Pedrini, Gaspar \& Sousa (2013), pode ser fomentado por propagandas, facilidades em conseguir receitas, alta taxa de dependência, entre outros. Vale ainda lembrar os perigos que o consumo irregular desses medicamentos pode causar, tais como: efeitos colaterais indesejados e dependência (Castro et al., 2013).

Ademais, entre os nossos respondentes, considerando aqueles que, atualmente, fazem uso de ansiolíticos, $25 \%$ deles iniciaram o consumo durante a pandemia de COVID-19. Isso demonstra uma associação do crescimento do uso de benzodiazepínicos à alta nas emoções relacionadas a ansiedade, problemas com o sono e adaptações necessárias durante esse período.

No que diz respeito às emoções, foram considerados sinais e sintomas de ansiedade e distúrbios do sono na realização do questionário de pesquisa. Dificuldade para iniciar o sono, insônia e/ou menos horas de sono foram apresentados por mais de $40 \%$ dos respondentes. Consequentemente, os números de cansaço irregular e sonolência também foram elevados, sendo $53,2 \%$ e $56,8 \%$, respectivamente.

Esses aspectos negativos em relação ao sono trazem uma associação à quantidade de respondentes que relata baixa concentração durante as aulas remotas, que somam $65,1 \%$ e pode ainda ser um dos motivos que estimule o consumo de ansiolíticos. Um estudo realizado em São Paulo com um grupo de mulheres que buscavam a atenção primária, mostra que a insônia ocupa o primeiro lugar $(48,14 \%)$ entre as motivações mais comuns para o uso de ansiolíticos benzodiazepínicos (Nordon, Akamine, Novo \& Hübner, 2009). Logo, os problemas de concentração apontados na pesquisa podem estar diretamente associados a questões com o sono e, nesse cenário, os ansiolíticos aparecem como uma solução - haja posta a sua utilização, também, como hipnóticos (ou indutores do sono, taxonomia mais atual).

No que concerne a sintomas físicos relacionados a ansiedade, tais como calafrios, falta de ar e palpitações irregulares, os números apresentados foram pouco significativos. Entretanto, os dados de medo iminente, insegurança e preocupação foram elevados, sendo este último presente em $77,1 \%$ dos estudantes. Esses resultados podem estar associados à manifestação da pandemia e a todas as necessidades de adaptação trazidas por ela. Considera-se ainda as taxas de "nervosismo", que aparece em $65,1 \%$, demonstrando sinais de estresse vividos em paralelo a essa nova reformulação de rotina e de vida.

Segundo Ozamiz-Etxebarria et al. (2020), já citados, em uma pesquisa realizada na Espanha, dados de progressão de sinais de estresse e ansiedade após o decreto de pandemia e estabelecimento do isolamento social apareceram com maior intensidade entre pessoas mais jovens. Os autores ainda 
associam a necessidade de adaptação ao modelo remoto de ensino como uma das causas do crescimento desses sintomas.

Esses dados estão ligados a respostas relacionadas a baixa adaptação ao modelo remoto de ensino entre os estudantes, que somam $45,8 \%$, e podem estar relacionados à necessidade de reformulação do ambiente doméstico para a inserção das atividades acadêmicas. Há ainda a transformação da rotina dos outros moradores da mesma casa, que agora compartilham o mesmo espaço para lazer, trabalho e descanso (Losekann \& Mourão, 2020).

Outra evidência que demonstra a dificuldade de gestão do tempo, mas também do espaço, se dá a partir dos $62 \%$ de estudantes respondentes que se sentiram sobrecarregados com as atividades acadêmicas. Nesse momento, aparecem baixos índices de concentração e motivação - dado este último presente em $73,4 \%$ dos estudantes. Em consequência disso, os números de diminuição de rendimento acadêmico $(41,2 \%)$ e pensamentos de trancamento de curso $(22,4 \%)$ crescem em consonância.

Os dados coletados na pesquisa, em geral, apontam sinais e sintomas de ansiedade, estresse e problemas com o sono que surgem durante a pandemia e afetam diretamente a relação dos estudantes participantes com o ambiente acadêmico - considerações que dialogam com nossas perguntas de investigação, apresentadas no início deste estudo, e que permitem afirmar, com considerável segurança, que tem havido agravos à saúde mental de estudantes universitários em decorrência da vivência acadêmica no período da pandemia ora vivenciada; incorrendo-se, adicionalmente, no aumento do consumo de ansiolíticos benzodiazepínicos. Além da pandemia, que perpassa por questões sérias de saúde e causa, por si só, medo do contágio e efeitos psicossociais da COVID-19 (Fundação Oswaldo Cruz, 2020), o ambiente acadêmico passou por transformações nunca antes vistas, de maneira rápida e que impactou diretamente a vida dos universitários.

\section{CONCLUSÃO}

Considerando-se os efeitos do ensino remoto na vivência acadêmica, este estudo sugere a necessidade de ser levado em consideração não apenas a diferença do método remoto, quando comparado ao método presencial. A mudança de método, necessitando de ajustes e capacidades de adaptação por parte de docentes e discentes universitários, é algo percebido e autorreferido como agravante da saúde mental, o que tem apontado - e aqui comprovamos - para um aumento do consumo de ansiolíticos benzodiazepínicos, princípio ativo já largamente consumido em nosso país, e que agora se apresenta com ainda mais força.

O aumento do número de sinais e sintomas - o que metaforiza um possível aumento do sofrimento psíquico na população estudada, em termos das ansiedades e dos problemas associados ao ciclo sono-vigília - impõe a necessidade de que instituições de ensino superior (IES) planejem ações de qualificação das ações ofertadas, mas também ações de incentivo ao cuidado e à oferta de espaços de salubridade mental durante a pandemia. Não se espera, vale ressaltar, que as próprias IES sejam capazes de ofertar ações em saúde (o que poderia descaracterizar a sua função precípua); mas espera-se que estas sejam capazes de articular ações com entidades da área da saúde (e não apenas em uma perspectiva curativa, mas sobretudo preventiva e promotora de saúde) que possam ofertar estratégias eficazes de autocuidado e produção de saúde mental, inclusive em ambiente doméstico.

Desconsiderar estes achados, bem como estas sugestões, podem significar que teremos estudantes cada vez mais em situações de sofrimento que lhe prejudiquem o desenvolvimento acadêmico - o que pode resultar em adoecimento, conflitos nas relações interpessoais e evasão do ambiente universitário. Em mesma medida, considerar estes achados significa também estimular a produção de pesquisas neste interim, especialmente dentro de cursos de graduação na área da saúde, em que podemos tomar a matéria real e cotidiana como elemento/instrumento de 
facilitação/mediação dos processos de ensino e aprendizagem, contribuindo com a produção de um conhecimento que reflita sobre o cotidiano e desenvolva tecnologias em saúde para a qualificação das ofertas de cuidado.

Como limites do estudo, cumpre-nos sinalizar todos os vieses passíveis de produção a partir de pesquisas on-line, em que o respondente não se encontra diretamente com o pesquisador. Muito embora reconheçamos estes limites, contudo, compreendemos que os mesmos abrem um flanco interessante de pesquisas que podem ser produzidas a partir de tal, como pesquisas que utilizem-se de metodologias mais qualitativas, investigando, em caráter mais aprofundado, como tem sido a relação dos estudantes universitários com o ambiente acadêmico, especialmente aqueles e aquelas que tenham iniciado a sua graduação em formato presencial e tenham transitado para o formato remoto, no início da pandemia; ou ainda aqueles e aquelas que, neste momento, estejam transitando de graduações iniciadas remotamente para aulas em formato presencial, haja posto discretos arrefecimentos da pandemia. Estudar os impactos subjetivos da vivência universitária neste momento, tal e qual o lugar social que a Universidade pode ocupar frente à pandemia em tema, nos parece um interessantíssimo horizonte. Ambos os achados podem nos subsidiar com boas informações para pensarmos em estratégias saudáveis de desenvolvimento de nossos ambientes acadêmicos, seja em contexto pandêmico ou pós-pandêmico.

Por fim, reconhecemos que o cenário vigente, perpassado pela pandemia da COVID-19, impõe desafios que potencializam aqueles já experienciados por estudantes universitários, ao cumprir com os seus deveres acadêmicos. Por isto mesmo, os sentimentos negativos associados a estas experiências, expressos na forma de ansiedade e alterações do ciclo sono-vigília, bem como no uso (não necessariamente responsável) de medicamentos psiquiátricos, por exemplo, podem ser associados à falta de ações efetivas acerca desta questão - lacuna em que consideramos, doravante, que este estudo tenha contribuído consideravelmente, e estimulamos que pesquisas outras futuras possam desenvolver-se, no sentido de ampliar os dados aqui retratados.

\section{REFERÊNCIAS}

Almondes, K. M., \& Araújo, J. F. (2003). Padrão do ciclo sono-vigília e sua relação com a ansiedade em estudantes universitários. Estudos de Psicologia (Natal), 8(1), 37-43. https://doi.org/10.1590/S1413-294X2003000100005

American Psychiatric Association. (2013). Diagnostic and Statistical Manual of Mental Disorders, Fifth Edition. Washington: APA.

Carvalho, M., Junqueira, L., Cerdeira, C., Costa, A., \& Santos, G.. (2017). Levantamento da Situação de Saúde Mental e Uso de Ansiolíticos e Antidepressivos por Acadêmicos do Curso de Odontologia de uma Universidade do Sul de Minas Gerais. Revista da Universidade Vale do Rio Verde. 15, 489-496. http://dx.doi.org/10.5892/ruvrd.v15i1.2772

Castioni, R., Melo, A. A. S., Nascimento, P. M., \& Ramos, D. L. (2021). Universidades federais na pandemia da Covid19: acesso discente à internet e ensino remoto emergencial. Ensaio: Avaliação e Políticas Públicas em Educação, Epub February 22, 2021. https://doi.org/10.1590/s0104-40362021002903108

Castro, G. L. G., Mendes, C. M. M., Pedrini, A. C. R., Gaspar, D. S. M., Souza, F. C. F. S. (2013). Uso de Benzodiazepínicos como automedicação: consequências do uso abusivo, dependência, farmacovigilância e farmacoepidemiologia. Revista Interdisciplinar, 6(1), 112-123. http://dx.doi.org/10.17648\%2F21

Fávero, V. R., Sato, M. del O., \& Santiago, R. M. (2017). Uso de Ansiolíticos: Abuso ou Necessidade?. Visão Acadêmica, 18(4), 98-106. https://revistas.ufpr.br/academica/article/view/57820

Ferreira, A. M. dos S., Príncipe, F., Pereira, H., Oliveira, I., \& Mota, L. (2020). COVimpact: pandemia COVID-19 nos estudantes do ensino superior da saúde. Revista De Investigação \& Inovação Em Saúde, 3(1), 7-16. https://doi.org/10.37914/riis.v3i1.80

Ferreira, C. L., Almondes, K. M., Braga, L. P., Mata, A. N. S., Lemos, C. A., \& Maia, E. M. C. (2009). Universidade, contexto ansiogênico? Avaliação de traço e estado de ansiedade em estudantes do ciclo básico. Ciência \& Saúde Coletiva, 14(3), 973-981. https://dx.doi.org/10.1590/S1413-81232009000300033 
Fundação Oswaldo Cruz. (2020). Saúde Mental e Atenção Psicossocial na Pandemia COVID-19. Boletim On-line da Fundação Oswaldo Cruz (FIOCRUZ). https://portal.fiocruz.br/sites/portal.fiocruz.br/files/documentos/cartilha_recomendacoes_gerais_06_04.pdf

Gil, A. C. (1987). Métodos e técnicas de pesquisa social. São Paulo: Atlas.

Leão, A. M., Gomes, I. P., Ferreira, M. J. M., \& Cavalcanti, L. P. G. (2018). Prevalência e Fatores Associados à Depressão e Ansiedade entre Estudantes Universitários da Área da Saúde de um Grande Centro Urbano do Nordeste do Brasil. Revista Brasileira de Educação Médica, 42(4), 55-65. https://doi.org/10.1590/1981$\underline{52712015 \mathrm{v} 42 \mathrm{n} 4 \mathrm{rb} 20180092}$

Losekann, R. G. C. B., \& Mourão, H. C. (2020). Desafios do Teletrabalho na Pandemia COVID-19: Quando o Home vira Office. Caderno de Administração, 28, 71-75. https://doi.org/10.4025/cadadm.v28i0.53637

Maia, B. R., \& Dias, P. C. (2020). Ansiedade, depressão e estresse em estudantes universitários: o impacto da COVID19. Estudos de Psicologia (Campinas), 37, e200067. Epub May 18, 2020. https://doi.org/10.1590/1982$\underline{0275202037 \mathrm{e} 200067}$

Malta, D. C., Szwarcwald, C. L., Barros, M. B. A., Gomes, C. S., Machado, I. E., Souza Júnior, P. R. B., Romero, D. E., Lima, M. G., Damacena, G. N., Pina, M. F., Freitas, M. I. F., Werneck, A. O., Silva, D. R. P., Azevedo, L. O., \& Gracie, R. (2020). A pandemia da COVID-19 e as mudanças no estilo de vida dos brasileiros adultos: um estudo transversal. Epidemiologia e Serviços de Saúde, 29(4), e2020407. Epub September 25, 2020. https://dx.doi.org/10.1590/s1679-49742020000400026

Marchi, K. C., Bárbaro, A. M., Miasso, A. I., \& Tirapelli, C. R. (2013). Ansiedade e consumo de ansiolíticos entre estudantes de enfermagem de uma universidade pública. Revista Eletrônica De Enfermagem, 15(3), 729-737. https://doi.org/10.5216/ree.v15i3.18924

Miranda, C. C. S., Lima, H. R. S., Alves, N. V. S., Sousa, L. L. D. A., Sousa, E. de S., Alves, K. K. S., Camapum, T. R. T., Santos, B. do S. de O., Santos, G. V. B. dos, Pereira, N. de A., \& Gonçalves, R. J. S. (2021). O Uso de Ansiolíticos e Antidepressivos entre Graduandos da Área da Saúde de Teresina-PI: Uma Pesquisa de Opinião. Revista de Casos e Consultoria, 12(1), e24679. https://periodicos.ufrn.br/casoseconsultoria/article/view/24679

Neri, J. V. D., Teston, A. P. M., \& Araújo, D. C. de M. (2020). Uso de Ansiolíticos e Antidepressivos por Acadêmicos da Área da Saúde: Uma Revisão Bibliográfica. Brazilian Journal Of Development, 6(10), 75673-75686. https://doi.org/10.34117/bjdv6n10-118

Nordon, D. G., Akamine, K., Novo, N. F., \& Hübner, C. K. (2009). Características do uso de benzodiazepínicos por mulheres que buscavam tratamento na atenção primária. Revista de Psiquiatria do Rio Grande do Sul, 31(3), 152-158. https://doi.org/10.1590/S0101-81082009000300004

Ozamiz-Etxebarria, N., Dosil-Santamaria, M., Picaza-Gorrochategui, M., \& Idoiaga-Mondragon, N. (2020). Stress, anxiety, and depression levels in the initial stage of the COVID-19 outbreak in a population sample in the northern Spain. Cadernos de Saúde Pública, 36(4), e00054020. Epub April 30, 2020. https://doi.org/10.1590/0102-311x00054020

Pereira, D. S., Souza, R. S., Buaiz, V., \& Siqueira, M. M. (2008). Uso de substâncias psicoativas entre universitários de medicina da Universidade Federal do Espírito Santo. Jornal Brasileiro de Psiquiatria, 57(3), 188195. https://doi.org/10.1590/S0047-20852008000300006

Portaria $N^{o} .343$ de 17 de março de 2020. (2020). Ministério da Educação. Diário Oficial da União. https://abmes.org.br/legislacoes/detalhe/3017/portaria-mec-n-343

Sue, V. M., \& Ritter, L. A. (2007). Conducting online surveys. Los Angeles: Sage Publications.

Souza, M. S. P., Almeida, R. L. M. de L., Amorim, A. T., \& Santos, T. A. dos. (2021). Uso de Antidepressivos e Ansiolíticos entre Estudantes do Curso de Farmácia em uma Instituição Privada e uma Pública do Interior da Bahia. Research, Society and Development, 10(8), e29610817177. http://dx.doi.org/10.33448/rsd-v10i8.17177

Zorzanelli, R. T., Giordani, F., Guaraldo, L., Matos, G. C., Brito Junior, A. G., Oliveira, M. G., Souza, R. M., Mota, R. Q. M., \& Rozenfeld, S. (2019). Consumo do benzodiazepínico clonazepam (Rivotril@) no estado do Rio de Janeiro, Brasil, 2009-2013: estudo ecológico. Ciência \& Saúde Coletiva, 24(8), 3129-3140. Epub August 05, 2019. https://doi.org/10.1590/1413-81232018248.23232017 